\title{
Demonstration of stable long-term operation of a kilohertz laser-plasma accelerator
}

\author{
L. Rovige $\odot,{ }^{1,}{ }^{*}$ J. Huijts $\odot,{ }^{1}$ I. Andriyash, ${ }^{1}$ A. Vernier, ${ }^{1}$ V. Tomkus $\odot,{ }^{2}$ V. Girdauskas, ${ }^{2,3}$ \\ G. Raciukaitis, ${ }^{2}$ J. Dudutis $\odot,{ }^{2}$ V. Stankevic $\odot,{ }^{2}$ P. Gecys $\odot,{ }^{2}$ M. Ouille $\odot,{ }^{1}$ Z. Cheng, ${ }^{1}$ \\ R. Lopez-Martens $\odot,{ }^{1}$ and J. Faure ${ }^{1}$ \\ ${ }^{1}$ LOA, CNRS, École Polytechnique, ENSTA Paris, Institut Polytechnique de Paris, Palaiseau, France \\ ${ }^{2}$ Center for Physical Sciences and Technology, Savanoriu Ave. 231, LT-02300, Vilnius, Lithuania \\ ${ }^{3}$ Vytautas Magnus University, K.Donelaicio St. 58. LT-44248, Kaunas, Lithuania
}

(Received 8 May 2020; accepted 24 August 2020; published 14 September 2020)

\begin{abstract}
We report on the stable and continuous operation of a kilohertz laser-plasma accelerator. Electron bunches with $2.6 \mathrm{pC}$ charge and $2.5 \mathrm{MeV}$ peak energy were generated via injection and trapping in a downward plasma density ramp. This density transition was produced in a specially designed asymmetrically shocked gas jet. The reproducibility of the electron source was also assessed over a period of a week and found to be satisfactory with similar values of the beam charge and energy. Particle in cell simulations confirm the role of the shock and the density transition in the electron injection mechanism. These results show that the reproducibility and stability of the laser-plasma accelerator are greatly enhanced on the long-term scale when using a robust scheme for density gradient injection.
\end{abstract}

DOI: 10.1103/PhysRevAccelBeams.23.093401

\section{INTRODUCTION}

Laser-plasma wakefield acceleration [1] enables the generation and acceleration of electrons beams over very short distances due to their extreme longitudinal accelerating fields, orders of magnitude higher than in conventional accelerators. When driven by $100 \mathrm{TW}$ to PW scale laser systems, laser-plasma accelerators (LPA) can produce electron beams in the $100 \mathrm{MeV}-\mathrm{GeV}$ energy range and are being considered as drivers for femtosecond $\mathrm{x}$-ray beams, either via betatron radiation [2], Compton scattering $[3,4]$, undulator radiation [5] or free electron laser radiation. Such femtosecond x-ray beams could enable time-resolved (pump-probe) experiments based on e.g., x-ray diffraction or spectroscopy. A more recent line of research is currently focusing on the development of high-repetition rate $(100 \mathrm{~Hz}-\mathrm{kHz})$ LPAs producing lower energy beams and requiring more modest laser parameters. TW-scale and kilohertz lasers with few-mJ pulse energy are capable of generating few $\mathrm{MeV}, \mathrm{pC}$ range electrons beams [6-9] with femtosecond durations. Such beams could be used for lowenergy applications such as ultrafast electron diffraction $[10,11]$ or irradiation of biological samples $[12,13]$.

\footnotetext{
*lucas.rovige@ensta-paris.fr

Published by the American Physical Society under the terms of the Creative Commons Attribution 4.0 International license. Further distribution of this work must maintain attribution to the author(s) and the published article's title, journal citation, and DOI.
}

In general, applications of LPAs require highly stable accelerator performances and the capacity to operate continuously and reliably over long periods of time, while providing consistent beam parameters from one day to the next. While numerous articles report on LPA stability on short timescales (as in a short series of shots over minutes) [14-16], there is a lack of consistent data in the literature addressing the issue of long-term stability and reproducibility. This is presumably because most published studies have focused on proof-of-principle experiments and on studying the physics of various regimes of plasma acceleration. This is now changing with initiatives such as the LUX beamline at DESY, aiming at turning a LPA into an actual machine [17]. In Ref. [17], day-long operation at a few $\mathrm{Hz}$ repetition rate is reported. However, to the best of our knowledge, no data has actually been published.

Concerning $\mathrm{kHz}$ laser-plasma accelerators, results obtained in the resonant condition for the bubble regime [6,7] yielded electron beams with $\mathrm{pC}$ charge in the $\mathrm{MeV}$ range. These experiments have reported on short term stability of the electron beam distribution but long-term stability has remained a challenge so far. This is because the $\mathrm{kHz}$ laser systems currently used to drive high-repetition LPAs have parameters that are at the limit of what is necessary to efficiently inject and accelerate electrons in the wakefield. In previous experiments, electron beam generation relied on ionization injection $[6,18,19]$ and self-injection [7]. Both mechanisms, being rather sensitive to the laser intensity, made electron injection in the wakefield difficult to control and stabilize, resulting in a high 
sensitivity to experimental parameters such as laser intensity, pulse duration, and small laser misalignments. Therefore, long-term stability has been difficult to achieve in this regime.

On the other hand, controlled injection techniques are known to increase the reliability of electron injection and, consequently, beam stability [14]. In particular, injection in a plasma density-transition [20-24] is relatively straightforward to implement experimentally. While the laser pulse goes through the density transition, the plasma wavelength increases so that the bubble is rapidly elongated, causing an effective slowing down of its back that facilitates the trapping of electrons. The concept of downward density ramp injection was validated in proof-of-principle experiments $[25,26]$. Other experiments relying on shock-front injection have also been successful [27-29]. In these experiments, a sharp density transition is obtained by inserting a knife-edge into the supersonic flow of the gas jet. These results indicate that density transition injection consistently triggers localized injection, and could provide the necessary stability for long-term and reproducible operation of a kilohertz laser-plasma accelerator.

In this paper, we report on the continuous and autonomous operation over 5 hours of a kilohertz LPA, generating $\mathrm{MeV}$-pC range electron beams thanks to the use of a newly designed asymmetrically shocked supersonic gas jet allowing injection in a sharp downward density gradient. The paper is organized as follows: in Sec. II, we first describe the experimental apparatus and in particular the gas jet design for generating a density transition. In Sec. III we show the result of $5 \mathrm{~h}$ operation and day-to-day reproducibility. The injection process is then investigated with particle-in-cell simulations. Finally, we conclude in Sec. IV.

\section{EXPERIMENTAL METHODS}

\section{A. One-sided shock nozzle}

Previous implementation of the density transition injection scheme relied on the lateral insertion of a knife-edge after the exit of the jet in order to induce a shock-front in the gas flow $[16,27]$. We propose a similar method, but the formation of the shock-front is directly incorporated in the design of the nozzle, which consists of a $100 \mu \mathrm{m}$ throat and $300 \mu \mathrm{m}$ exit diameter "De Laval" nozzle to which a $100 \mu \mathrm{m}$ long flat section has been added at the end of one side of the nozzle (see Fig. 1(a), [30]). These nozzles will thereafter be referred to as "one-sided shock" (OSS) nozzles. The flat section induces an abrupt change of direction in the gas flow which, due to the flow being supersonic, translates into a shock-front formation and therefore leads to a sharp density gradient downstream [31]. Compared to inserting a knife edge into the jet, incorporating the shock-front generation directly in the nozzle design offers a solution that is more robust and easier to implement on such a small scale. However, machining such

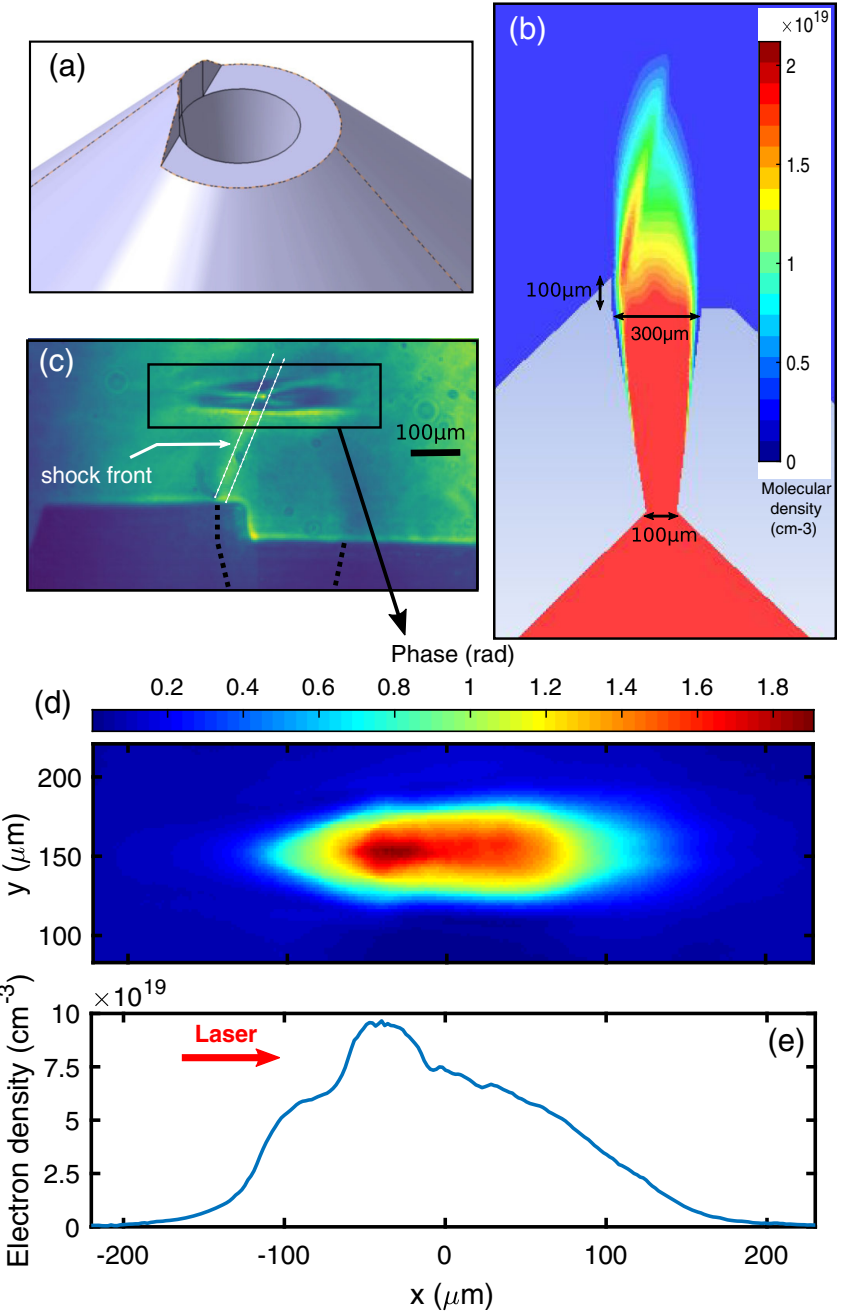

FIG. 1. (a) 3D model of the one-sided shock nozzle. (b) Molecular density map from a 3D CFD simulation of an OSS jet performed with Fluent. Gas is $\mathrm{N}_{2}$ and backing pressure $\mathrm{P}=20$ Bar. Color map is capped at $2.1 \times 10^{19} \mathrm{~cm}^{-3}$ for viewing purpose. (c) Shadow graphic side view image of the plasma. Black dotted line suggests the inner walls of the nozzle, white dotted lines follow the shock front. (d) Experimental phase from the plasma column measured with the wave-front sensor. The $y$-axis represents the distance from the nozzle exit, the $x$-axis is the laser propagation axis. (e) Electronic density profile at $150 \mu \mathrm{m}$ from the OSS nozzle's exit, retrieved by Abel inversion of the phase map. Laser propagation direction is from left to right (red arrow).

a complicated nozzle geometry, including micrometer scale features, is technologically demanding. In practice, the nozzle was made using nanosecond laser rear-side processing and the femtosecond laser-assisted selective etching technique (FLSE, [32,33]) which consists of chemical etching of previously selectively laser-irradiated fused silica. 3D computational fluid dynamics (CFD) simulations were realized with the software ANSYS Fluent to validate and optimize the design. Figure 1(b) shows a map of the molecular density obtained by simulating a nitrogen flow 
through the OSS nozzle, with a backing pressure of 20 bar. The shock-front in gas density originating from the final straight section is clearly visible.

The plasma density profile was characterized experimentally by sending the laser pulse into a nitrogen gas jet produced from the OSS nozzle. The plasma column produced by the main beam was illuminated from the side by a probe beam, and imaged on a quadriwave lateral shearing interferometer (SID4-HR, Phasics, $[34,35]$ ). The plasma density profile can be derived from the phase maps [see Fig. 1(d)] via Abel inversion, assuming radial symmetry around the horizontal axis. Figure 1(e) shows the measured electron density line out at $150 \mu \mathrm{m}$ from the nozzle exit, with a backing pressure of 22 bar. The peak density is $9.7 \times 10^{19} \mathrm{~cm}^{-3}$ and the density after the shock is $7.3 \times$ $10^{19} \mathrm{~cm}^{-3}$, corresponding to a $25 \%$ density drop with a transition width of $15 \mu \mathrm{m}$. Note that with nitrogen, a single molecule of $\mathrm{N}_{2}$ produces 10 electrons once ionized by the laser to $\mathrm{N}^{5+}$, which provides the high plasma density required to resonantly drive the plasma wakefield, while keeping the background pressure in the vacuum chamber to a reasonable level and therefore making continuous kilohertz operation possible. Finally, in this free flowing jet, the stability of the plasma density is determined by the precision of the pressure regulator, and is estimated to be better than $4 \%$.

\section{B. Experimental setup}

The experiment was conducted using the Salle Noire laser system at LOA [36,37], which provides $10 \mathrm{~mJ}, 25 \mathrm{fs}$
FWHM laser pulses at kilohertz repetition rate, with a central wavelength $\lambda_{0}=800 \mathrm{~nm}$. The laser pulse is then post-compressed in a helium-filled hollow core fiber (HCF) resulting in a broad spectrum, $4.0 \mathrm{fs}$ pulse. Through a pair of motorized fused silica wedges we can control the amount of dispersion to fine-tune pulse compression or add chirp to the pulse, and measure the pulse temporal intensity profile using the d-scan technique [38]. The energy on target was $3.8 \mathrm{~mJ}$ and the pulses are focused by a f/4 off-axis parabola, resulting in a $6.2 \mu \mathrm{m} \times 5.5 \mu \mathrm{m}$ FWHM focal spot, which corresponds to a Rayleigh range of $z_{R} \sim 100 \mu \mathrm{m}$. These laser parameters yield a measured peak intensity in vacuum of $\mathrm{I}=2.0 \times 10^{18} \mathrm{~W} \mathrm{~cm}^{-2}$ and a normalized vector potential $a_{0} \simeq 1.0$. The charge as well as the electron beam distribution were measured with a calibrated $\mathrm{CsI}(\mathrm{Tl})$ phosphor screen imaged onto a high dynamic range CCD camera. The laser beam was blocked by a thin aluminium foil in front of the phosphor screen, so as to block electrons with energies below $100 \mathrm{keV}$. The energy of the electrons was measured with a retractable spectrometer made of a $500 \mu \mathrm{m}$ pinhole and two permanent cylindrical magnets.

It is important to note that the laser-plasma accelerator truly runs at a repetition rate of $1 \mathrm{kHz}$ : the gas jet flows continuously while the pumping system is able to maintain a vacuum of a few $10^{-3}$ mbar; the data was continuously collected during the experimental run, often by acquiring data accumulated over several shots. In order to perform a long run it was necessary to implement numerous

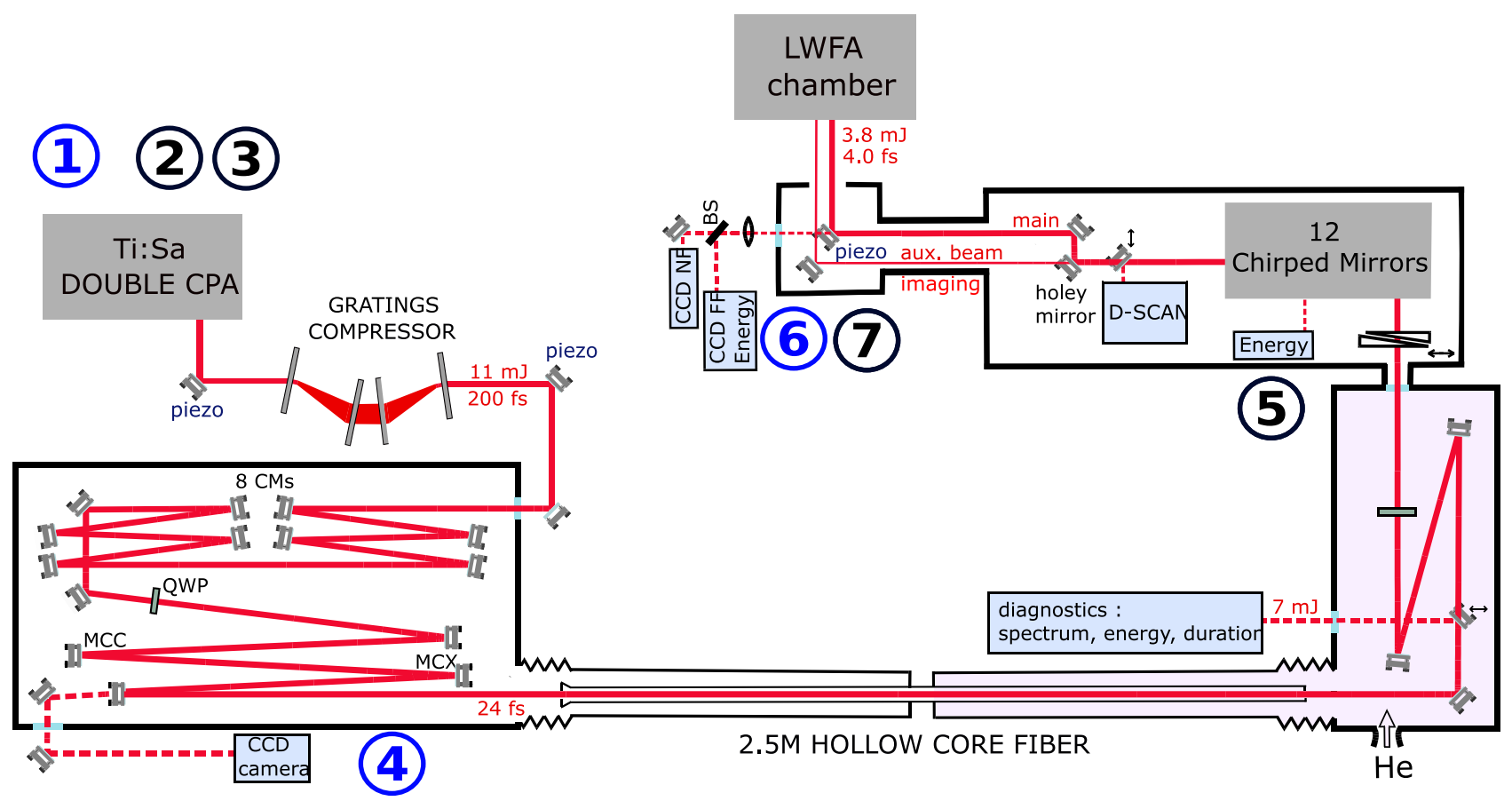

FIG. 2. Schematic of the laser system. Numbers (1),(4,(6) show the position of the beam pointing stabilization devices, numbers (2),(3),(5,(7) correspond to the position of energy diagnostics for the booster amplifier, the power amplifier, the hollow core fiber (HCF) compressor and the turning box, respectively. 
diagnostics and feedback loops in the laser system to ensure that the laser parameters remained stable over a long time. Figure 2 shows a schematic of the laser system with indicated positions where laser energy monitoring was performed. In order to maximize the stability of the energy, the spectrum, and the spatial mode output of the $\mathrm{HCF}$, a fast beam pointing system is essential. Considering the long optical path from the exit of the fiber to the experiment itself, compensation for long-term thermal drifts proved crucial to the long-term stability of the LPA. We thus set up a slow beam pointing device operating at $<1 \mathrm{~Hz}$ just before the experiment, using the leakage through of mirror in the turning box just before the final focusing parabola-point (7) in Fig. 2. This ensured that the laser beam alignment on the gas jet stayed rigorously the same and that the focal spot quality was identical throughout the long acquisition run. Images of the focal spot were taken before and after the experiment, and displayed no significant evolution.

\section{RESULTS}

\section{A. Stability over several hours of continuous operation}

The beam profile and charge were measured right before the start of the electron spectrum monitoring. Statistics were performed from 20 acquisitions, each consisting of an accumulation over 10 shots, thus accounting for 200 shots in total. This initial measurement yielded a mean charge of $2.6 \mathrm{pC}$ per shot with a $0.6 \mathrm{pC}$ standard deviation, and a beam divergence of $80 \times 75 \mathrm{mrad} \pm 8 \times 9 \mathrm{mrad}$ FWHM [see Fig. 3(c)]. Electron beam pointing stability is $17 \times 11 \mathrm{mrad}$ RMS. This regime was obtained by setting the focal point of the laser at the position of the shock-front, strongly suggesting that the injection occurs indeed at the density transition. The electron spectrum was then monitored during 5 hours of complete hands-off operation of the kilohertz laser-plasma accelerator, i.e., with no other intervention than the beam pointing stabilization feedback loops at the three above-mentioned locations in the laser chain, see Fig. 2. Results of this measurement are displayed in Fig. 3(a). Beams with peaked spectra and a large majority of electrons with energy exceeding $1 \mathrm{MeV}$ were reliably produced throughout the whole 306 min of monitoring. Moreover, during the first $150 \mathrm{~min}$, the spectrum remained very stable, with a peak energy of $2.5 \mathrm{MeV}$. After that, the high-energy part noticeably eroded with time, lowering the peak energy to $1.9 \mathrm{MeV}$. Possible reasons for this energy loss will be addressed later in this article. A comparison of the spectra during the first $150 \mathrm{~min}$ and the last $50 \mathrm{~min}$ is represented in Fig. 3(b).

We emphasize that this level of stability was only obtained with the OSS nozzles. Indeed, during the same experimental campaign, we also tested several De Laval supersonic jets with no shock and similar dimensions (300 $\mu \mathrm{m}$ exit diameter). Without the shock, no stable production of electrons with a peak at $2-3 \mathrm{MeV}$ could be achieved on the 10 minute scale, and the source performance was more sensitive to slight drifts of laser position or alignment.

To complete these data and assess more thoroughly the question of stability, we plot the temporal evolution of the

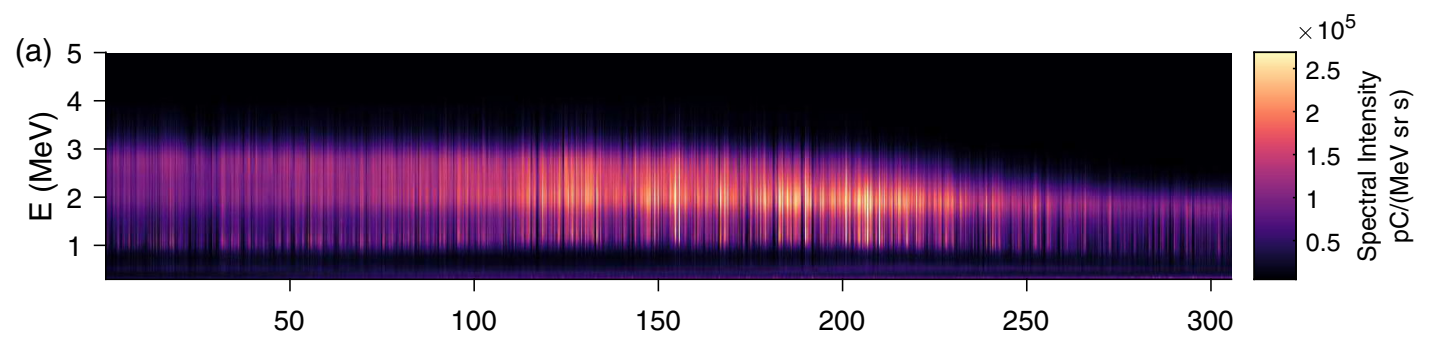

(b)

Time (min)
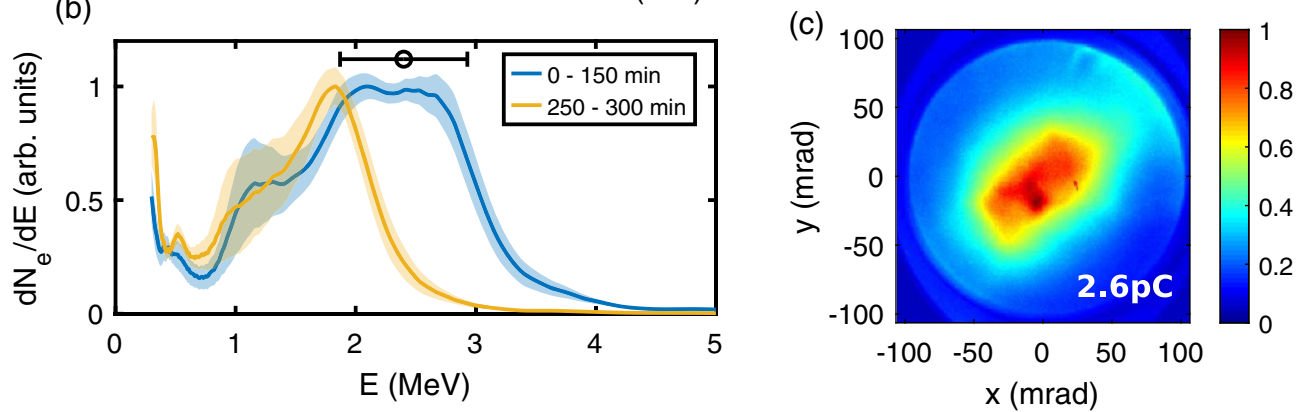

FIG. 3. (a) Electron spectra measured continuously for 306 min. Each spectrum is averaged over 100 shots. (b) Electron spectra averaged over 0-150 min (blue) and 250-300 min (orange), and their standard deviations (shaded area); the black error bar represents the spectrometer resolution at $2.4 \mathrm{MeV}$. (c) Electron beam measured just before the start of the $5 \mathrm{~h}$ spectrum monitoring. The total charge per shot is $2.6 \mathrm{pC} \pm 0.6 \mathrm{pC}$ (std). The beam divergence is approximately 80 mrad FWHM. 

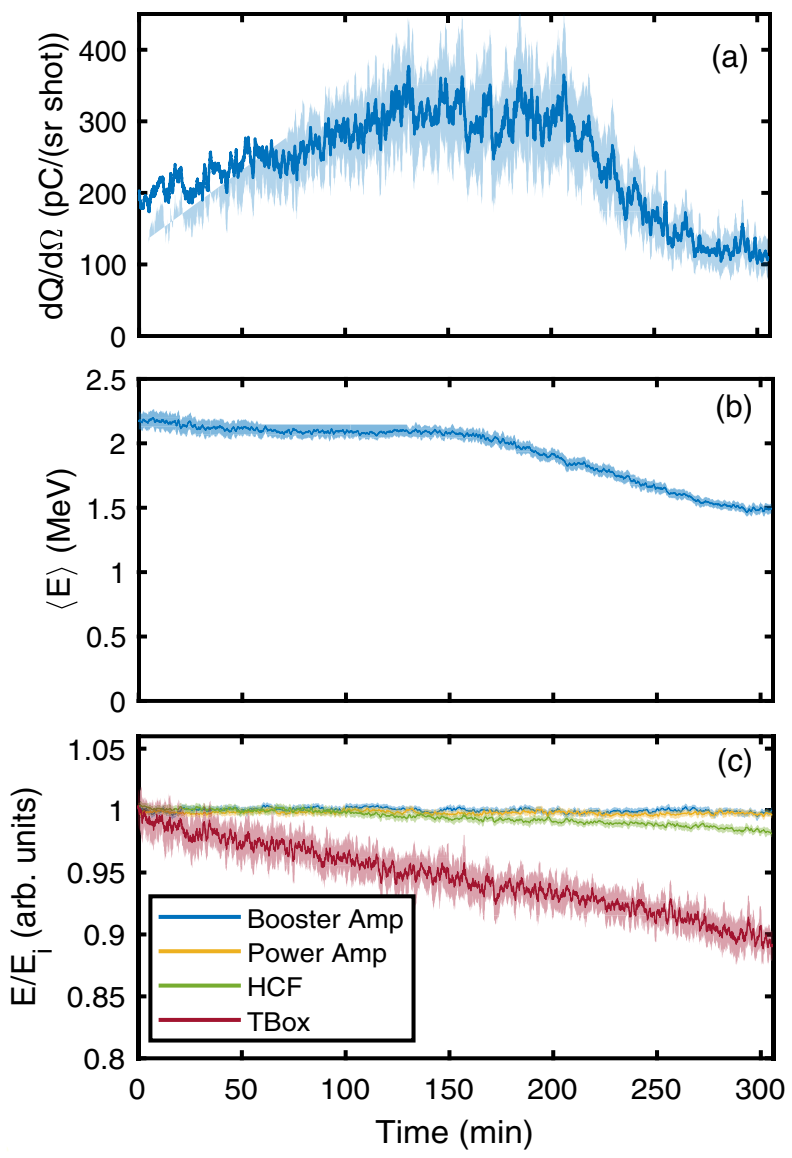

FIG. 4. (a) Total charge per solid angle collected through the electron spectrometer pinhole. (b) Mean electron beam energy versus time. (c) Monitoring of the laser energy at four points in the laser chain. Each curve in this figure is averaged over a one minute moving window and shaded areas represent the corresponding standard deviation.

total charge per solid angle $d Q / d \Omega$ collected through the pinhole of the spectrometer [Fig. 4(a)], the mean energy of the electrons $\langle E\rangle$ [Fig. 4(b)], as well as the relative laser energy at different points of the laser chain [Fig. 4(c)]. All curves are averaged over a 1 min moving window. The data show an increase in $d Q / d \Omega$ [Fig. 4(a)] during the first $130 \mathrm{~min}$. This is likely due to a small angular drift of the electron beam on a long time scale, resulting in a higher electron signal through the electron spectrometer pinhole. Therefore, only the short term variation of the charge can be estimated from this measurement, giving typical fluctuation of about $50 \mathrm{pC} /(\mathrm{sr}$ shot $)$ corresponding to $20 \%$ RMS.

Figure 4(b) confirms the observations made previously regarding the stability of the spectrum, and indeed, shows that the mean energy of the electrons is quite stable at $\langle E\rangle \simeq 2.1 \mathrm{MeV}$ during the first $150 \mathrm{~min}$ of monitoring, with short-term RMS variations of only $2-4 \%$ [shaded area in Fig. 4(b)]. The decrease of the mean beam energy to $\langle E\rangle \simeq 1.5 \mathrm{MeV}$ toward the end of the run can also be clearly observed. Note that during the run, the laser system

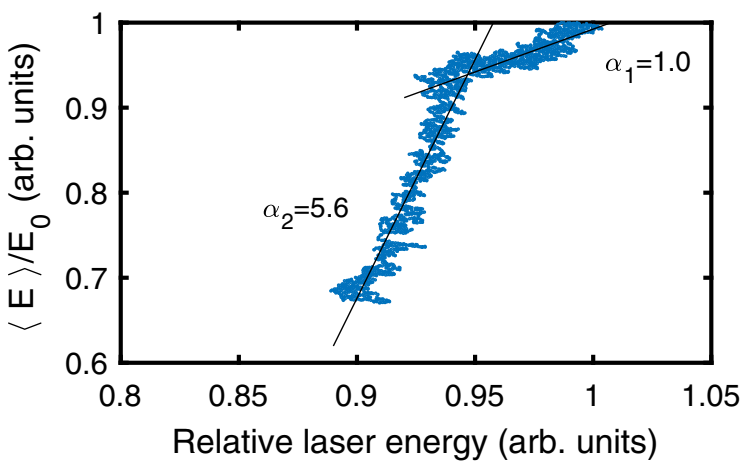

FIG. 5. Correlation between the electron mean energy and the relative laser energy right before the off-axis focusing parabola, and linear fits of the two different parts of the curve, with slopes $\alpha_{1}$ and $\alpha_{2}$.

was extremely stable, see Fig. 4(c), except for the energy measured using the turning box diagnostic [red curve in Fig. 4(c)], which is the last measurement point before the focusing parabola and is, therefore, the most representative of the evolution of the laser energy on target. The energy measured at this point decreased steadily during the experiment and reached a $11 \%$ relative loss after 306 min. It is likely that this progressive energy loss was due to the slow damage of a few chirped mirrors at the end of the compressor. The damage on the mirrors was found during the inspection which we performed the day after the experiment. Interestingly, the evolution of the electron energy can be correlated to the evolution of the laser energy at this last measurement point. To display these correlations, Fig. 5 shows the normalized mean energy of the electrons plotted against the laser relative energy. Two different correlation regimes are clearly distinguishable: (i) the first $5 \%$ of laser energy loss leads to a 5\% energy loss of the electrons suggesting a linear correlation. We then observe a threshold effect, (ii) as the next 5\% drop of laser energy correlates with a $30 \%$ electron mean energy loss. Assuming a linear dependence in both regimes, the two parts of the correlation plot are linearly fitted, yielding a slope $\alpha_{1}=1.0$ in the first five percents of energy loss, and a slope $\alpha_{2}=5.6$ in the following five percents. This highlights the importance of laser energy stability: in our case, energy variations larger than $5 \%$ can cause significant modifications of the electron spectrum due to what seems like a threshold effect. Nevertheless, decent stability of the electron beam was achieved over the 300 min of continuous operation, with the first $150 \mathrm{~min}$ period displaying a remarkable stability correlated to the highest laser performance. This is a significant improvement compared to previous results of our group where this level of stability would not be achieved on times longer than 10 minutes. Stability over multiple hours of operation with $\mathrm{pC}-\mathrm{MeV}$ range electron beams at kilohertz repetition rate, corresponding to more than $18 \times 10^{6}$ consecutive shots, 
TABLE I. Various experimental parameters and electron beam performance showing slight variations from day to day but overall fair reproducibility of the experiment. The values after the \pm sign are RMS deviation.

\begin{tabular}{lccc}
\hline \hline & Day 1 & Day 2 & Day 3 \\
\hline $\mathrm{I}\left(\mathrm{W} \mathrm{cm}^{-2}\right)$ & $1.8 \times 10^{18}$ & $2.0 \times 10^{18}$ & $1.6 \times 10^{18}$ \\
$\mathrm{n}_{\text {e,peak }}\left(\mathrm{cm}^{-3}\right)$ & $8.8 \times 10^{19}$ & $9.7 \times 10^{19}$ & $9.7 \times 10^{19}$ \\
$\mathrm{Q}(\mathrm{pC} / \mathrm{shot})$ & $1.6 \pm 0.2$ & $2.6 \pm 0.6$ & $1.4 \pm 0.2$ \\
div. fwhm $(\mathrm{mrad})$ & $42 \pm 10$ & $77 \pm 7$ & $57 \pm 11$ \\
$\langle\mathrm{E}\rangle(\mathrm{MeV})$ & $2.29 \pm 0.13$ & $2.11 \pm 0.06$ & $2.19 \pm 0.04$ \\
\hline \hline
\end{tabular}

represents significant progress toward scientific applications of laser-plasma accelerators.

\section{B. Day to day reproducibility}

In order to determine the reproducibility of the electron beam and to assess the sensitivity of the accelerator to small day-to-day variations of the laser parameters, the measurements were repeated on three different days, each separated by about a week. The same actual OSS nozzle was used for the three experimental runs and showed no degradation over time. Table I summarizes experimental conditions for each day, as well as the charge and mean electron energy corresponding to the electrons spectra displayed in Fig. 6. These results show that the downward gradient injection method with one-sided shock nozzles increased significantly the reliability of the accelerator. Indeed, electrons beams with similar charge and 2-3 MeV peaked spectrum were easily obtained even though the experimental parameters varied slightly from day to day. In particular, we see that experiments from day 2 and day 3, performed at the same plasma density, yield very similar electron spectra. We emphasize that this level of reproducibility was not observed using more conventional supersonic jets in our previous experiments [9]. Such level of reproducibility is also decisive for a reliable use of the accelerator for

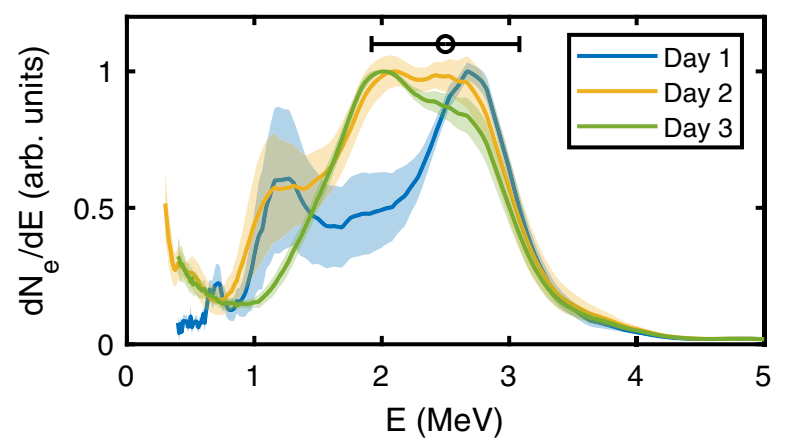

FIG. 6. Electron spectra obtained on three different days with the same one-sided shock nozzle. Spectra are the results of averaging over 2000 shots for day 1 and day 2, and 5000 shots for day 3. Day 1 and day 2 are 7 days apart, day 2 and day 3 are 6 days apart. applications. Moreover, these fused-silica nozzles showed a great resilience to damage, as the one used for this experiment provided reliable and reproducible results even after using it for about $30-50 \times 10^{6}$ shots.

\section{Simulations}

To gain insight into the injection process and the role of the density transition, we now turn to particle-in-cell (PIC) simulations. The modeling was done with the spectral quasi-3D code FBPIC [39], using a cylindrical simulation domain with the grid size $\Delta z=26 \mathrm{~nm}, \Delta r=2 \Delta z, 5$ azimuthal Fourier modes, and 16 macroparticles of initially neutral nitrogen per cell. We paid special attention to using parameters as close as possible to the experiment and we injected in the PIC simulation the measured laser intensity profile at focus and the measured temporal field maps. To produce the realistic laser pulse, we have used the intensity profile and the temporal field map recorded experimentally at the laser focus. First, this field is produced in the simulation domain using the antenna-a 2D array of macroparticles, which oscillate and generate self-consistently all the components of the desired electromagnetic field. Next, we reconstruct the converging out-of-focus beam by modeling its in-vacuum propagation for $140 \mu \mathrm{m}$ in the backward direction (negative z-direction). Finally, for the LWFA modeling, the obtained pulse is launched in the forwards direction into the plasma. The plasma density profile was also taken from the measurement of Fig. 1(e).

In Fig. 7(a) we plot the spectral distributions of electrons produced from the L-shell and K-shell of nitrogen atoms. Here we consider only electrons whose energies are larger than $>300 \mathrm{keV}$ and final divergence angles smaller than $100 \mathrm{mrad}$ aperture (as in the experiment). Evidently, $\mathrm{K}$-shell electrons, originating from the ionization of $\mathrm{N}^{5+}$ ions, form the population of electrons injected by ionization. The total charge in the simulation is $4.4 \mathrm{pC}$ with a large energy peak at $\sim 3 \mathrm{MeV}$, in good agreement with experiments. However, electrons from ionization injection form a population of electrons above $4 \mathrm{MeV}$ [green curve in Fig. 7(a)] and represent $20 \%$ of the charge. In the experiment, these energetic particles, $>4 \mathrm{MeV}$ were not observed, indicating that ionization injection might be slightly overestimated in the simulation. Note that ionization injection below the barrier suppression ionization regime is likely to the overestimated for two reasons: (i) the ADK tunnel ionization model [40] employed in the simulation and (ii) the very high sensitivity to laser intensity in the tunneling regime. Despite this minor difference, the spectrum of L-shell electrons and the angular distribution (shown in the inset), agree well with experimental results. In Fig. 7(b), blue and red curves show the dynamics of electron injection and laser peak field as it propagates in the plasma (gray area). Figure 7(b) shows that electrons are injected as the laser propagates through the density down-ramp confirming the importance of the 

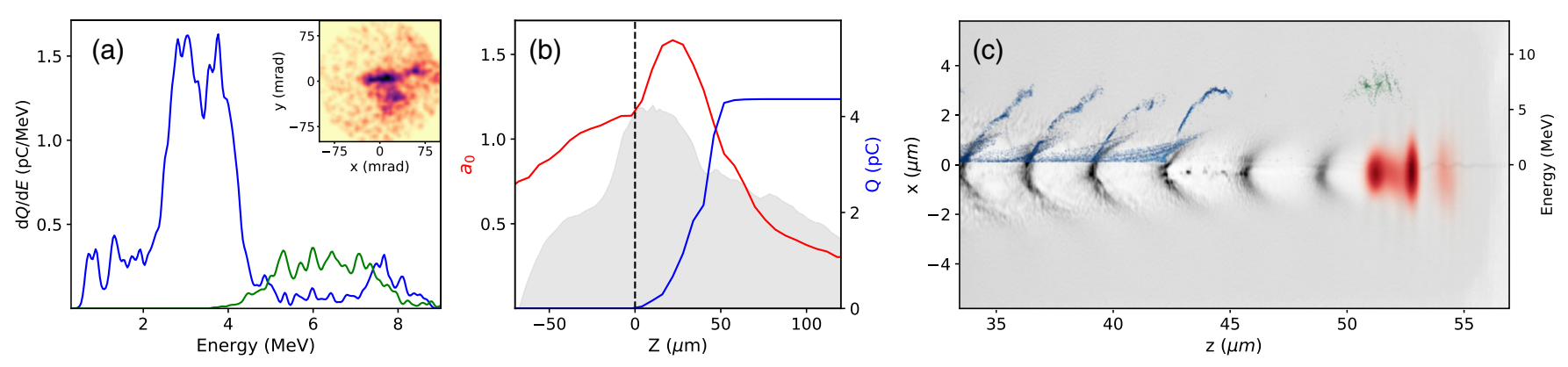

FIG. 7. PIC simulations. (a) Final energy spectra of electrons produced from L-shell (blue curve) and K-shell (green curve) of nitrogen atoms, with energies $>300 \mathrm{keV}$ and within $100 \mathrm{mrad}$ aperture; electron angular distribution (inset); (b) Evolution of the peak normalized laser field $e E_{l} / m_{e} c \omega_{0}$, and electron charge of the beam selected from (a); gray area represents the adjusted plasma density profile in arbitrary units. (c) Electron density (gray color) in $z-x$ plane, and in phase space $z-\varepsilon_{z}$ for L-shell and K-shell electrons (blue and green respectively) and 2D laser envelope (red color).

density transition for triggering electron injection. Note that the laser also undergoes self-focusing as it propagates in the shocked region indicating that the increase of laser intensity also enhances electron injection. Finally, a series of simulations at different laser focus positions, $z_{\mathrm{foc}} \in(0,40) \mu \mathrm{m}$, was performed, showing that injection always occurs in the vicinity of the shock and in the density down-ramp. This suggests that the density transition region stabilizes the injection process by localizing it to the shocked region.

In Fig. 7(c), we plot the plasma density, phase distribution of electrons, and on-axis laser field at the end of density down-ramp, when L-shell electrons (in blue) have reached the decelerating phase of the wakefield. One can see that K-shell electrons (in green) originating from ionization injection are located in the first bucket, i.e., in the region of strong laser field, as expected. On the other hand, L-shell electrons are trapped in multiple buckets following the second bucket. This behavior can be explained by considering the wake phase velocity in a density gradient (cf. [24]):

$$
v_{p}=c \frac{1}{1+(z-c t) \frac{1}{k_{p}} \frac{d k_{p}}{d z}},
$$

where $k_{p}=\sqrt{4 \pi r_{e} n_{e}}$ is the plasma wave number that varies with local plasma density $n_{e}(z)$, and where $r_{e}$ is the classical electron radius. For the down-ramp profile, the local $v_{p}$ decreases in the comoving frame of the laser with the comoving coordinate $(z-c t)$. Therefore, the wake phase velocity is smaller in buckets that are further away from the laser pulse and trapping of slower electrons is facilitated in these buckets. A similar effect has been observed experimentally in [41], when a laser was focused at the exit ramp of a subsonic jet. From this analysis, we conclude that shorter and possibly higher quality electron bunches might be obtained by localizing the injection in the first bucket of the wake. Following Eq. (1), this might be achieved by further lowering $v_{p}$ using a steeper gradient (larger $d k_{p} / d z$ ), or by increasing the energy of plasma electrons with a higher laser intensity.

\section{CONCLUSION}

In conclusion, by using an asymmetrically shocked fusedsilica nozzle, we were able to produce the sharp density transition and the high plasma density necessary for downward density gradient injection in a LPA driven by few-cycle laser pulses. We managed to stabilize the injection and obtained electron beams with picocoulomb charge and $\mathrm{MeV}$ peaked spectrum for 5 hours of continuous operation. Good day-to-day reproducibility was also demonstrated. In addition, the electron energy was correlated to the laser energy stability, showing that energy fluctuations should remain at the percent level in order to ensure stable accelerator operation. This level of performance could allow users to perform low-energy radiobiology experiments with significant irradiation dose and an acceptable $20 \%$ charge stability. Similarly, the source performance is adapted to irradiation experiments for testing the radiation hardness of space electronics [42]. Concerning application to electron diffraction, the energy spread of $60 \%$ is still too large as such experiments typically require energy spreads around the percent level. Nevertheless, this requirement could be met by using a narrow energy filter, coupled with an electron beam line such as the one suggested in [11] to improve the emittance. Still, further experimental efforts have to be made in order to increase the quality of the electron beam, with higher charge and higher energy, while maintaining a high level of stability. Using helium instead of nitrogen could limit ionization defocusing of the laser, thus enabling higher intensities and subsequently higher electron energies (although pumping helium will require a more powerful pumping system to support $\mathrm{kHz}$ operation). Another possible way to improve the energy of our LPA could be to use even smaller OSS jets, more adapted to the acceleration scale, in order to avoid electron dephasing (this will also be a challenge for micronozzle manufacturing). Finally, PIC simulations confirmed the role of the density transition in 
the localization of electron injection and suggest that sharper gradients and higher laser intensities should permit to concentrate the charge in the first bucket, thereby producing few femtosecond bunches. These issues will be tackled in future work.

\section{ACKNOWLEDGMENTS}

This work was funded by the European Research Council (ERC Starting Grant FEMTOELEC) under Contract No. 306708. Financial support from the Région Île-deFrance (under Contract No. SESAME-2012-ATTOLITE) and the Extreme Light Infrastructure-Hungary Non-Profit Ltd (under Contract No. NLO3.6LOA) is gratefully acknowledged. We also acknowledge Laserlab-Europe, Grant No. H2020 EC-GA 654148 and the Lithuanian Research Council under Grant agreement No. S-MIP-17-79.

[1] T. Tajima and J. M. Dawson, Laser Electron Accelerator, Phys. Rev. Lett. 43, 267 (1979).

[2] A. Rousse, K. T. Phuoc, R. Shah, A. Pukhov, E. Lefebvre, V. Malka, S. Kiselev, F. Burgy, J.-P. Rousseau, D. Umstadter, and D. Hulin, Production of a keV X-Ray Beam from Synchrotron Radiation in Relativistic LaserPlasma Interaction, Phys. Rev. Lett. 93, 135005 (2004).

[3] K. T. Phuoc, S. Corde, C. Thaury, V. Malka, A. Tafzi, J. P. Goddet, R. C. Shah, S. Sebban, and A. Rousse, All-optical compton gamma-ray source, Nat. Photonics 6, 308 (2012).

[4] S. Chen, N. D. Powers, I. Ghebregziabher, C. M. Maharjan, C. Liu, G. Golovin, S. Banerjee, J. Zhang, N. Cunningham, A. Moorti, S. Clarke, S. Pozzi, and D. P. Umstadter, MevEnergy X Rays from Inverse Compton Scattering with Laser-Wakefield Accelerated Electrons, Phys. Rev. Lett. 110, 155003 (2013).

[5] M. Fuchs, R. Weingartner, A. Popp, Z. Major, S. Becker, J. Osterhoff, I. Cortrie, B. Zeitler, R. Hörlein, G. D. Tsakiris, U. Schramm, T. P. Rowlands-Rees, S. M. Hooker, D. Habs, F. Krausz, S. Karsch, and F. Grüner, Laser-driven soft-X-ray undulator source, Nat. Phys. 5, 826 (2009).

[6] D. Guénot, D. Gustas, A. Vernier, B. Beaurepaire, F. Böhle, M. Bocoum, M. Lozano, A. Jullien, R. Lopez-Martens, A. Lifschitz, and J. Faure, Relativistic electron beams driven by khz single-cycle light pulses, Nat. Photonics 11 (2017).

[7] D. Gustas, D. Guénot, A. Vernier, S. Dutt, F. Böhle, R. Lopez-Martens, A. Lifschitz, and J. Faure, High-charge relativistic electron bunches from a khz laser-plasma accelerator, Phys. Rev. Accel. Beams 21, 013401 (2018).

[8] F. Salehi, A. J. Goers, G. A. Hine, L. Feder, D. Kuk, B. Miao, D. Woodbury, K. Y. Kim, and H. M. Milchberg, Mev electron acceleration at $1 \mathrm{khz}$ with $<10 \mathrm{mj}$ laser pulses, Opt. Lett. 42, 215 (2017).

[9] J. Faure, D. Gustas, D. Guénot, A. Vernier, F. Böhle, M. Ouillé, S. Haessler, R. Lopez-Martens, and A. Lifschitz, A review of recent progress on laser-plasma acceleration at $\mathrm{kHz}$ repetition rate, Plasma Phys. Controlled Fusion 61, 014012 (2018).
[10] Z.-H. He, A. G. R. Thomas, B. Beaurepaire, J. A. Nees, B. Hou, V. Malka, K. Krushelnick, and J. Faure, Electron diffraction using ultrafast electron bunches from a laserwakefield accelerator at $\mathrm{khz}$ repetition rate, Appl. Phys. Lett. 102, 064104 (2013).

[11] J. Faure, B. van der Geer, B. Beaurepaire, G. Gallé, A. Vernier, and A. Lifschitz, Concept of a laser-plasmabased electron source for sub-10-fs electron diffraction, Phys. Rev. Accel. Beams 19, 021302 (2016).

[12] O. Rigaud, N. O. Fortunel, P. Vaigot, E. Cadio, M. T. Martin, O. Lundh, J. Faure, C. Rechatin, V. Malka, and Y. A. Gauduel, Exploring ultrashort high-energy electron-induced damage in human carcinoma cells, Cell Death and Disease 1, e73 (2010).

[13] O. Lundh, C. Rechatin, J. Faure, A. Ben-Ismaill, J. Lim, C. De Wagter, W. De Neve, and V. Malka, Comparison of measured with calculated dose distribution from a 120-mev electron beam from a laser-plasma accelerator, Med. Phys. 39, 3501 (2012).

[14] J. Faure, C. Rechatin, A. Norlin, A. Lifschitz, Y. Glinec, and V. Malka, Controlled injection and acceleration of electrons in plasma wakefields by colliding laser pulses, Nature (London) 444, 737 (2006).

[15] J. Osterhoff, A. Popp, Z. Major, B. Marx, T. P. RowlandsRees, M. Fuchs, R. Hörlein, F. Grüner, D. Habs, F. Krausz, S. M. Hooker, and S. Karsch, Stable laserdriven electron beams from a steady-state-flow gas cell, AIP Conf. Proc. 1086, 125 (2009).

[16] A. Buck, J. Wenz, J. Xu, K. Khrennikov, K. Schmid, M. Heigoldt, J. M. Mikhailova, M. Geissler, B. Shen, F. Krausz, S. Karsch, and L. Veisz, Shock-Front Injector for High-Quality Laser-Plasma Acceleration, Phys. Rev. Lett. 110, 185006 (2013).

[17] N. Delbos, C. Werle, I. Dornmair, T. Eichner, L. Hübner, S. Jalas, S. Jolly, M. Kirchen, V. Leroux, P. Messner, M. Schnepp, M. Trunk, P. Walker, P. Winkler, and A. Maier, Lux-a laser-plasma driven undulator beamline, Nucl. Instrum. Methods Phys. Res., Sect. A 909, 318 (2018), 3rd European Advanced Accelerator Concepts workshop (EAAC2017).

[18] A. Pak, K. A. Marsh, S. F. Martins, W. Lu, W. B. Mori, and C. Joshi, Injection and Trapping of Tunnel-Ionized Electrons into Laser-Produced Wakes, Phys. Rev. Lett. 104, 025003 (2010).

[19] C. McGuffey, A. G. R. Thomas, W. Schumaker, T. Matsuoka, V. Chvykov, F. J. Dollar, G. Kalintchenko, V. Yanovsky, A. Maksimchuk, K. Krushelnick, V. Y. Bychenkov, I. V. Glazyrin, and A. V. Karpeev, Ionization Induced Trapping in a Laser Wakefield Accelerator, Phys. Rev. Lett. 104, 025004 (2010).

[20] S. Bulanov, N. Naumova, F. Pegoraro, and J. Sakai, Particle injection into the wave acceleration phase due to nonlinear wake wave breaking, Phys. Rev. E 58, R5257 (1998).

[21] P. Tomassini, M. Galimberti, A. Giulietti, D. Giulietti, L. A. Gizzi, L. Labate, and F. Pegoraro, Production of highquality electron beams in numerical experiments of laser wakefield acceleration with longitudinal wave breaking, Phys. Rev. Accel. Beams 6, 121301 (2003). 
[22] J. U. Kim, N. Hafz, and H. Suk, Electron trapping and acceleration across a parabolic plasma density profile, Phys. Rev. E 69, 026409 (2004).

[23] H. Suk, H. J. Lee, and I. S. Ko, Generation of high-energy electrons by a femtosecond terawatt laser propagating through a sharp downward density transition, J. Opt. Soc. Am. B 21, 1391 (2004).

[24] A. V. Brantov, T. Z. Esirkepov, M. Kando, H. Kotaki, V. Y. Bychenkov, and S. V. Bulanov, Controlled electron injection into the wake wave using plasma density inhomogeneity, Phys. Plasmas 15, 073111 (2008).

[25] C. G. R. Geddes, K. Nakamura, G. R. Plateau, C. Toth, E. Cormier-Michel, E. Esarey, C. B. Schroeder, J. R. Cary, and W. P. Leemans, Plasma-Density-Gradient Injection of Low Absolute-Momentum-Spread Electron Bunches, Phys. Rev. Lett. 100, 215004 (2008).

[26] J. Faure, C. Rechatin, O. Lundh, L. Ammoura, and V. Malka, Injection and acceleration of quasimonoenergetic relativistic electron beams using density gradients at the edges of a plasma channel, Phys. Plasmas 17, 083107 (2010).

[27] K. Schmid, A. Buck, C. M. S. Sears, J. M. Mikhailova, R. Tautz, D. Herrmann, M. Geissler, F. Krausz, and L. Veisz, Density-transition based electron injector for laser driven wakefield accelerators, Phys. Rev. Accel. Beams 13, 091301 (2010).

[28] C. Thaury, E. Guillaume, A. Lifschitz, K. Ta Phuoc, M. Hansson, G. Grittani, J. Gautier, J.-P. Goddet, A. Tafzi, O. Lundh, and V. Malka, Shock assisted ionization injection in laser-plasma accelerators, Sci. Rep. 5, 16310 (2015).

[29] K. K. Swanson, H.-E. Tsai, S. K. Barber, R. Lehe, H.-S. Mao, S. Steinke, J. van Tilborg, K. Nakamura, C. G. R. Geddes, C. B. Schroeder, E. Esarey, and W. P. Leemans, Control of tunable, monoenergetic laser-plasma-accelerated electron beams using a shock-induced density downramp injector, Phys. Rev. Accel. Beams 20, 051301 (2017).

[30] See Supplemental Material at http://link.aps.org/ supplemental/10.1103/PhysRevAccelBeams.23.093401

for top-view image of a one-sided shock nozzle taken with an optical microscope.

[31] R.D. Zucker and O. Biblarz, Fundamentals of Gas Dynamics (John Wiley \& Sons, New York, 2002).

[32] A. Marcinkevičius, S. Juodkazis, M. Watanabe, M. Miwa, S. Matsuo, H. Misawa, and J. Nishii, Femtosecond laserassisted three-dimensional microfabrication in silica, Opt. Lett. 26, 277 (2001).
[33] V. Tomkus, V. Girdauskas, J. Dudutis, P. Gečys, V. Stankevič, and G. Račiukaitis, High-density gas capillary nozzles manufactured by hybrid 3d laser machining technique from fused silica, Opt. Express 26, 27965 (2018).

[34] J. Primot and L. Sogno, Achromatic three-wave (or more) lateral shearing interferometer, J. Opt. Soc. Am. A 12, 2679 (1995).

[35] J. Primot and N. Guérineau, Extended hartmann test based on the pseudoguiding property of a hartmann mask completed by a phase chessboard, Appl. Opt. 39, 5715 (2000).

[36] F. Böhle, M. Kretschmar, A. Jullien, M. Kovacs, M. Miranda, R. Romero, H. Crespo, U. Morgner, P. Simon, R. Lopez-Martens, and T. Nagy, Compression of CEPstable multi-mJ laser pulses down to 4 fs in long hollow fibers, Laser Phys. Lett. 11, 095401 (2014).

[37] M. Ouillé, A. Vernier, F. Böhle, M. Bocoum, A. Jullien, M. Lozano, J.-P. Rousseau, Z. Cheng, D. Gustas, A. Blumenstein, P. Simon, S. Haessler, J. Faure, T. Nagy, and R. Lopez-Martens, Relativistic-intensity near-singlecycle light waveforms at $\mathrm{khz}$ repetition rate, Light Sci. Appl. 9, 47 (2020).

[38] M. Miranda, C. L. Arnold, T. Fordell, F. Silva, B. Alonso, R. Weigand, A. L'Huillier, and H. Crespo, Characterization of broadband few-cycle laser pulses with the d-scan technique, Opt. Express 20, 18732 (2012).

[39] R. Lehe, M. Kirchen, I. A. Andriyash, B. B. Godfrey, and J.-L. Vay, A spectral, quasi-cylindrical and dispersion-free particle-in-cell algorithm, Comput. Phys. Commun. 203, 66 (2016).

[40] M. V. Ammosov, N. B. Delone, and V. P. Krainov, Tunnel ionization of complex atoms and atomic ions by an alternating electromagnetic field, Sov. Phys. JETP 64, 1191 (1986), http://www.jetp.ac.ru/cgi-bin/e/index/e/64/6/ p1191?a=list.

[41] B. Beaurepaire, A. Vernier, M. Bocoum, F. Böhle, A. Jullien, J.-P. Rousseau, T. Lefrou, D. Douillet, G. Iaquaniello, R. Lopez-Martens, A. Lifschitz, and J. Faure, Effect of the Laser Wave Front in a Laser-Plasma Accelerator, Phys. Rev. X 5, 031012 (2015).

[42] B. Hidding, O. Karger, T. Königstein, G. Pretzler, G. G. Manahan, P. McKenna, R. Gray, R. Wilson, S. M. Wiggins, G. H. Welsh, A. Beaton, P. Delinikolas, D. A. Jaroszynski, J. B. Rosenzweig, A. Karmakar, V. FerletCavrois, A. Costantino, M. Muschitiello, and E. Daly, Laser-plasma-based space radiation reproduction in the laboratory, Sci. Rep. 7, 42354 (2017). 